\title{
Analysis of Landslide Stability Based on the Morgenstern-Price Method
}

\author{
Qiang Fan ${ }^{*}$, Jiayang Lin, Wencheng Sun, Jie Lu and Peihua Chen \\ Southwest Branch of State Grid Corporation of China, Chengdu, Sichuan Province, China
}

\begin{abstract}
The landslide is a kind of natural disaster caused by slope instability. Due to its wide distribution, frequent occurrence, and damaging effects, many countries in the world suffer from it. Sichuan Province in China is a landslide-prone area since it locates in the seismic zone. In this paper, the Morgenstern-Price method is used to analyze the stability of a landslide in Sichuan Province, and heavy rainfall is also simulated to analyze the landslide stability under such conditions, which can provide assistance for geological disaster prevention in the future.
\end{abstract}

\section{Introduction}

The landslide, one of the most common natural disaster, is the downslope movement of rock and soil under gravity along a certain weak surface. It is caused by natural factors such as earthquakes and rainfall, as well as human activities. Due to their own instability, slopes often slide or collapse under the influenced of geology, topography and weather, which affects the surrounding roads, bridges and residential areas, and poses great threat to human life and property. For example, on 31 May, 1970, a major earthquake struck the top of Peruvian Andes. Millions of cubic meters of rock fell off the mountainside, triggering a rock collapse that moved $14 \mathrm{~km}$ within 3 minutes, buried a city and caused more than 25,000 deaths [1].

According to the statistics from China Institute of Geological Environment Monitoring(CIGEM), from 1995 to 2003, sudden geological disasters like landslides and mudslides across the country have caused a total of 10499 deaths and disappearances, 65356 injuries, and 57.5 billion Yuan in property loss. Among all the areas, Sichuan Province, with complete stratum lithology, complex geological structure and diverse geological environments, is one of the provinces with high risk of geological disasters. It also suffered from the "May 12" Wenchuan earthquake in 2008, which caused adverse effect on the geological environment in the earthquake areas. The geological disasters in this province are characterized by wide range, large scale, quick formation, high frequency and long duration. Fig. 1 shows a landslide in Leshan City, Sichuan Province, in 2018 with a volume of about 50,000 cubic meters [2]. Landslides can not only lead to direct casualties and economic losses but also cause further losses by impeding the traffic and rescue. On 20 August 2019, heavy rainfall caused flash floods and landslides in Wenchuan County, Li County, Maoxian County and Songpan County in Aba Autonomous Prefecture, Sichuan

*Corresponding author: 18628028963@126.com 
Province, resulting in 16 deaths and 22 disappearances, with direct economic losses of 3.6 billion Yuan. In addition to the impact of earthquake on the geological environment, the climate of the Sichuan Province is relatively complex. Sichuan is located in a transitional zone affected by a variety of monsoon circulations and its western region is prone to rainstorms and floods. The average annual precipitation of the province is $978.8 \mathrm{~mm}$, and $70 \%$ of it is concentrated from May to September, which is more likely to cause danger. Therefore, it is of great urgency to establish the corresponding landslide analysis model for Sichuan Province and take effective prevention.

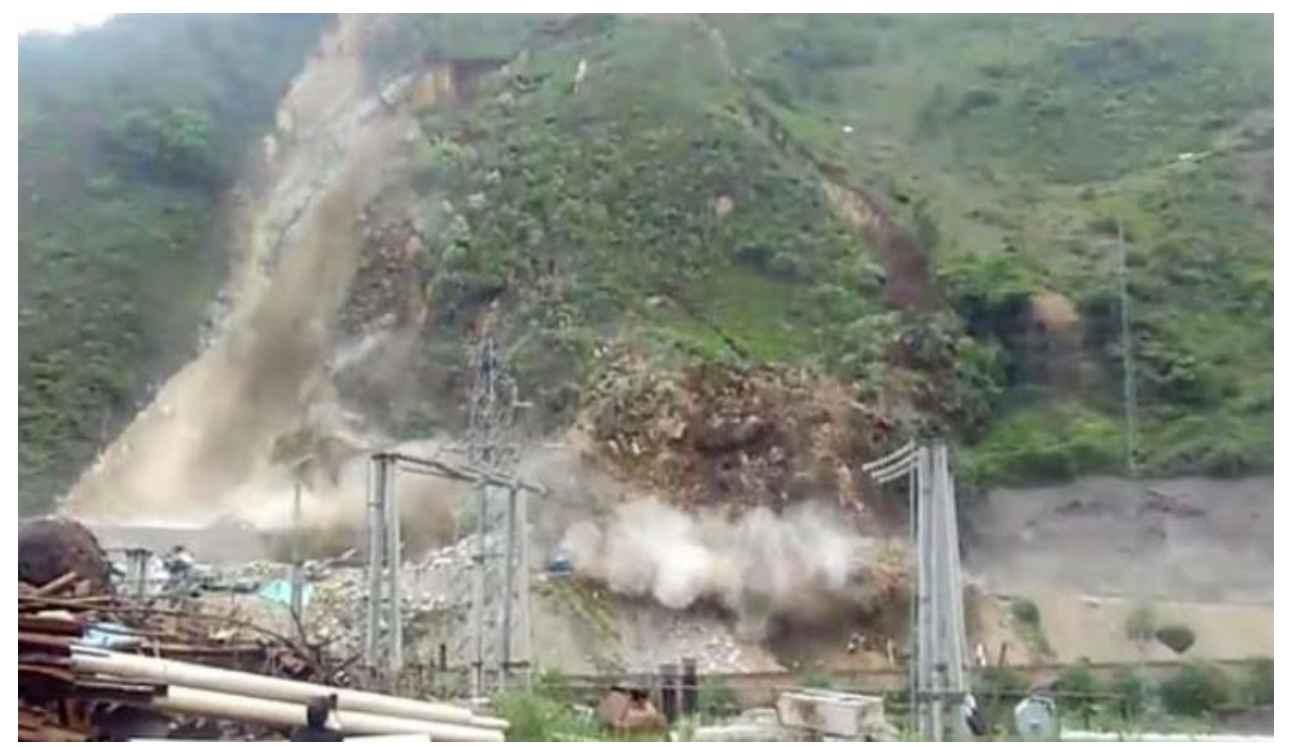

Fig. 1. Large-scale Landslide in Leshan City, Sichuan Province, China

For decades, the study of landslides has never stopped. Yu Kai researched the effects of rainfall intensity, rainfall duration and rainfall type on slope stability [3]. Chimidi used GIS statistical methods to evaluate and partition landslides in Jinbi Town, western Ethiopia based on nine factors, such as the slope material, elevation, gradient, aspect and curvature[4]. As for research method, there are the Ordinary Method which only considers the balance between the sliding moment and the anti-skid moment of the arc center but not the critical slip surface and the minimum safety factor [5], the GLE method which analyzes how the safety factors are related with the inclination of the forces between the rocks and the balance of the forces [6], and the Morgenstern-Price method (M-PM) which assumes that there is a functional relationship between the inter-bar tangential component and the normal component. [7].

In this paper, the Morgenstern-Price method was first used to analyze the slope stability of a certain landslide in Jiuzhaigou County, Aba Autonomous Prefecture, Sichuan province. Then the rainfall conditions of 48 hours before and after the rain storm disaster on August 20, 2019 was simulated to analyze the slope stability of the mountain structure under specific rainfall conditions.

\section{Methodology}

To understand the complicated mechanism of landslide formation, the basic conditions must be known first. A landslide hazard requires (1) loss of support in the mountain front, 
which provides enough space for the sliding of the mountain; (2) cut surface on both sides, which allows the soft soil layer to slide down; and (3) the weak strata. From the perspective of rock types, the soft rock group containing sand, gravel, and weathered crust has low shear strength and cannot withstand the influence of gravity and downward displacement deformation. Besides, the pressure of the trailing edge soil layer on the leading edge soil layer would also trigger landslides [8]. The experimental area is located in a district with high probability of heavy rainfall in summer, where the rock is glutenite. In this paper, the Morgenstern-Price method is firstly applied to calculate the stability or the safety factor F of the slope without rainfall, and then the slope stability is analyzed according to the rainfall situation in the area.

\subsection{Morgenstern-Price method}

The Morgenstern-Price method takes moment equilibrium into full consideration and therefore is a strict method for stability calculation. The static equilibrium differential equation is established as follow:

$$
-P(x)=\cos \left(\varphi_{e}^{\prime}-\alpha+\beta\right) \frac{d G}{d x}-\sin \left(\varphi_{e}^{\prime}-\alpha+\beta\right) \frac{d \beta}{d x} G
$$

In which:

$$
\begin{gathered}
P(x)=\left(\frac{d w}{d x}+q\right) \sin \left(\varphi_{e}^{\prime}-\alpha\right)-r_{u} \frac{d w}{d x} \sec (\alpha) \sin \left(\varphi_{e}^{\prime}\right)+C_{e}^{\prime} \sec (\alpha) \sin \left(\varphi_{e}^{\prime}\right)-\eta \frac{d w}{d x} \cos \left(\varphi_{e}^{\prime}-\alpha\right) \\
C_{e}^{\prime}=\frac{C^{\prime}}{F} \\
\varphi_{e}^{\prime}=\operatorname{arctg}\left(\frac{\operatorname{tg} \varphi^{\prime}}{F}\right)
\end{gathered}
$$

The moment equilibrium equation is:

$$
G \sin (\beta)=-y \frac{d}{d x}(G \cos (\beta))+\frac{d}{d x}\left(y_{t} G \cos (\beta)\right)+\eta \frac{d \omega}{d x} h_{t}(5)
$$

The boundary conditions are:

$$
\left.\begin{array}{c}
G(a)=0 \\
G(b)=0 \\
y_{t}(a)=y(a) \\
y_{t}(b)=y(b)
\end{array}\right\}
$$

Based on Equation (1), Equation (2) and boundary condition (6), the equilibrium formula of static force and moment can be expressed as: 


$$
\left.\begin{array}{c}
\int_{b}^{a} P(x) S(x) d x=0 \\
\int_{b}^{a} P(x) S(x) t(x) d x-M_{e}=0
\end{array}\right\}
$$

In Equation (7), $\mathrm{S}(\mathrm{x}), \mathrm{t}(\mathrm{x})$ and $\mathrm{M}_{\mathrm{e}}$ are expressed as follow:

$$
\begin{aligned}
& S(x)=\sec \left(\varphi_{e}^{\prime}-\alpha+\beta\right) e^{\int_{a}^{x} \operatorname{tg}\left(\varphi_{e}^{\prime}-\alpha+\beta\right) d \beta} \\
& t(x)=\int_{a}^{x}(\sin (\beta)-\cos (\beta) \operatorname{tg}(\alpha)) \exp ^{\int_{a}^{\xi} \operatorname{tg}\left(\varphi_{e}^{\prime}-\alpha+\beta\right) \frac{d \beta}{d \xi} d \xi} \\
& M_{e}=\int_{a}^{b} \eta \frac{d \omega}{d x} h_{1} d x
\end{aligned}
$$

Where, $\mathrm{F}$ is the safety factor, $\mathrm{C}^{\prime}$ and $\varphi^{\prime}$ are the effective values of rock cohesion and effective angle of internal friction respectively, $r_{u}$ is the pore water pressure coefficient, $W$ is the weight of the slice, $\eta$ is the seismic force coefficient, and $G$ is the total force on the vertical edge of the slice. Equation (7) reflects the static and moment balance requirements of sliding rock and soil. The safety factor $\mathrm{F}$ and the inclination angle $\beta(\mathrm{x})$ of the lateral force of the rocks along the way are included in the two equations. $\lambda$ and $F$ can be solved through Equation (11) .

$$
\operatorname{tg}(\beta)=\lambda f(x)
$$

\subsection{Slope stability analysis}

First, establish the landslide area in the seep/w module and define the landslide soil material in the area, and then define the head boundary of the groundwater. The third step is to set the entry and exit range of the sliding surface in the slope/w module to calculate the landslide safety factor. The safety factor of the slope stability $\mathrm{F}$ is the ratio of the antisliding force to the sliding force along the assumed slip surface. When the ratio is greater than 1 , the slope is stable; when it equals 1 , the slope is in a state of limit equilibrium; when it is less than 1, the slope is damaged. After the stability degree or safety factor $F$ is obtained, the slope stability is analyzed in the following two situations:

\subsubsection{In the absence of rainfall or earthquake.}

F is used as the standard to evaluate the slope stability. The smaller its value, the greater the possibility of landslide.

\subsubsection{Under rainfall conditions.}

As the rainfall continues and intensifies, the infiltration of rainwater into the soil mass of the accumulation layer can produce influences in three aspects. First, the rainfall will increase the hydrostatic pressure and hydrodynamic pressure of the slope, and the infiltration water will enter the cracks of rock and the pores of accumulation layer, which will soften the rock and soil by increasing its water content and bulk density and therefore 
decrease the shear resistance index of the rock and soil. Second, the infiltration water will raise the groundwater level and reduce the effective normal stress of the sliding surface. The seepage pressure will also increase the sliding force of the slope. Third, rainfall infiltrates into the bedrock surface or the water-breaking layer below the weathered rock and soil to become a lubricant, which reduces the sliding resistance of the contact surface and leads to landslides. The rainfall data used in this simulation was based on the station rainfall data for a total 48 hours from 0: 00 a.m. on August 19 to 0: 00 a.m. on August 21, 2019. And F is recalculated and used as the standard to evaluate the slope stability according to the simulated rainfall model combined with the landslide model. The smaller its value, the greater the possibility of landslides.

\section{Results and discussion}

\subsection{Experimental data}

Based on the above analysis, a landslide model was built in GeoStudio. By reference to the table of Implementation of Geological Hazard Prevention in Sichuan Province in 2020, only the rainfall factor was selected as the landslide inducing factor. The selected area is the third group of Punan Village, Wujiao Town, Jiuzhaigou County, Aba Prefecture, Sichuan Province, and the longitude and latitude coordinates are $104.2475^{\circ} \mathrm{E}$, $32.91166666666667^{\circ} \mathrm{N}$. The height and slope of the landslide are determined by the elevation and distance measurement, and then the corresponding material-free model was established. In consideration of the presence of perched water in the mountain and the existence of a debris layer of about 5 meters on the surface, the established model was divided into four regions as shown in Figure 2.

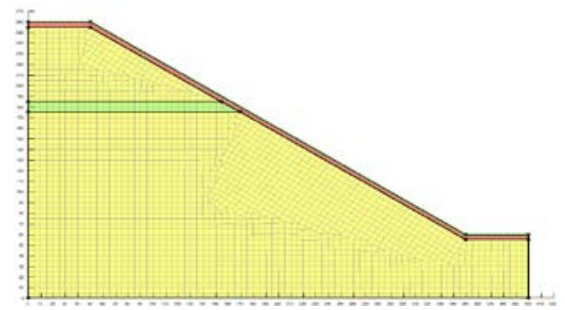

Fig. 2. Landslide Model

According to the 1:200,000 geological map I4827 (Sichuan Province) in the National Geological Archives of China(NGAC), the rock property of the landslide point was confirmed to be Xujiahe Formation of the Upper Triassic while the landslide material was glutenite. According to the table of Stratigraphic-lithologic Characteristics of the Sichuan Basin [9], the lithology is gray-white fine sandstone and medium-grained sandstone interbedded with black shale. According to the current National Rock Classification and Naming Scheme [10], the gray-white fine sandstone is mainly composed of fine sand and silt, with the fine sand being the majority, while the medium sandstone is medium sand mixed with coarse sand. Therefore, it can be assumed that the rocks in the study area range from fine sand to coarse sand, most of which is fine sand. According to the particle size grading of sedimentary rocks, the diameter of the particles is between $0.125 \mathrm{~mm}$ and $0.5 \mathrm{~mm}$. According to the reference values of soil permeability coefficient [11], the coefficient of saturated permeability is $0.504 \mathrm{~m} / \mathrm{h}$. The volumetric water content in the saturated soil is $0.35 \mathrm{~m}^{-3} / \mathrm{m}^{-3}$ [12]; the unite weight is $20.9 \mathrm{KN} / \mathrm{m}^{-3}$; the effective cohesion is $36.5 \mathrm{kpa}$; and the effective angle of friction is $20^{\circ}$. Finally, the buried depth of groundwater table was 
determined. According to the analysis that the rock property of the landslide point is the Xujiahe Formation of the Upper Triassic [13] and that the landslide point is located in the northwestern part of Sichuan Province [14], it can be known that the aquifer is composed of the sandstone of Cretaceous and Upper Triassic Xujiahe Formation interbedded with shale, mudstone and coal seam, which is thick and stable. It is generally expressed as confined groundwater, of which the initial water head is high and then decreases gradually. The buried depth of the confined aquifer roof is 24 to 40 meters. After confirming the parameters required by the relevant landslide model, the soil-water characteristic curve and the saturated permeability coefficient function are estimated, as shown in Figure 3 and Figure 4. The soil-water characteristic curve reflects the relationship between the volumetric water content of the soil and the suction of the matrix, and it is closely related to the soil aggregate structure, composition and porosity. The strength and permeability coefficient of unsaturated soil and the distribution of water above the groundwater level can be determined according to the soil-water characteristic curve, which is very important for the study of the transformation among saturated/unsaturated seepage field, volumetric water content and matric suction.

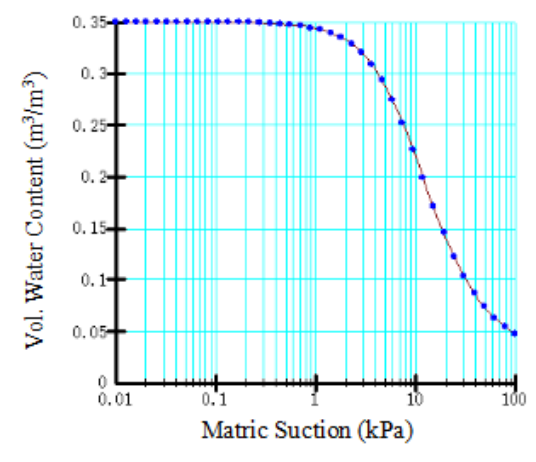

Fig. 3. Function of Soil-water Characteristic Curve.

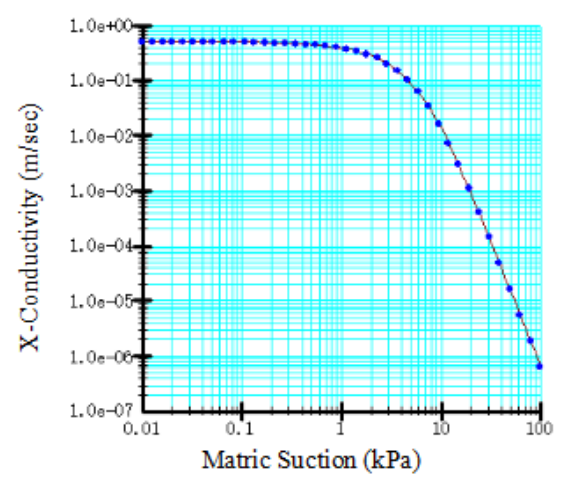

Fig. 4. Function of Permeability Coefficients.

Rainfall will cause changes in pore water pressure, thereby affecting the stability of slopes. Pore pressure is the pressure of groundwater in soil or rock, which acts between particles or pores. With the significant rises or falls of the groundwater level, the effective self-weight stress will increase or decrease accordingly, resulting in the changes of the pore pressure. When the groundwater level is relatively static, there is still a change in pore water pressure, which is caused by infiltration. And the pore pressure is determined by the height of capillary water rise. Negative pore water pressure refers to the pore pressure that is less than atmospheric pressure. Therefore, the analysis of landslides cannot be limited to those under static situations. In most cases, the slope instability is caused by rainfall or earthquakes on the landslide body. The research simulated the rainfall in 48 hours before and after the extreme rainstorm event on 20 August, 2019 and the simulated rainfall function is shown in Figure 5. The maximum hourly rainfall intensity is $28.6 \mathrm{~mm}$ at the $21 \mathrm{st}$ hour and second intensity is $24.3 \mathrm{~mm}$ at the 27 th hour. 


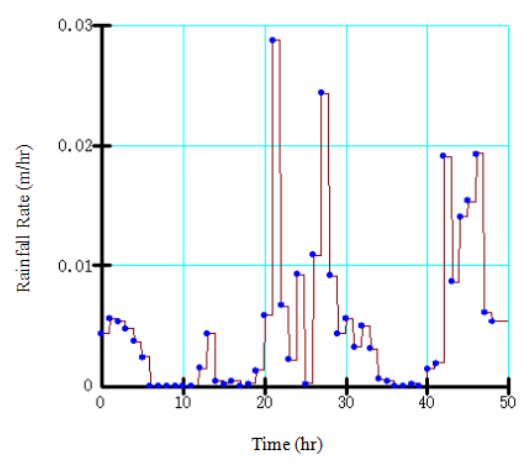

Fig. 5. Simulation of Precipitation.

\subsection{Discussion}

As shown in Figure 6, the static slope stability analysis is performed on the landslide model to analyze the potential sliding surface and slope safety factor. As the slope safety factor is 0.755 , less than 1 , the mountain is at risk of landslides.

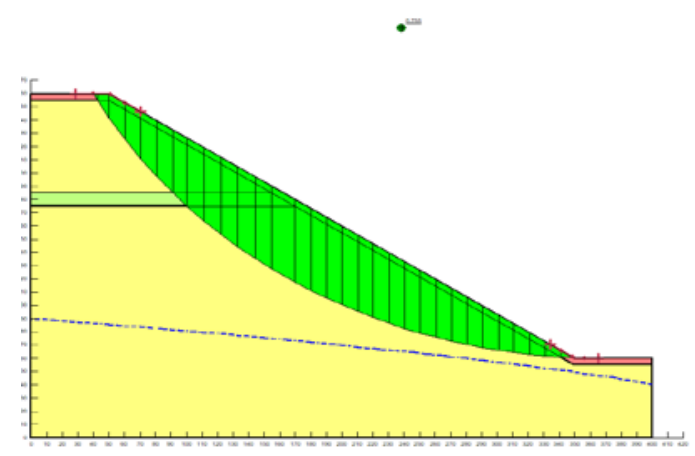

Fig. 6. Slope Stability Analysis Under Static Conditions.

It can be seen from the rainfall boundary conditions that the rainfall reached the peaks at the 21st hour and 27th hour. The figures below show the distribution of pore pressure in the landslide model at the 1st, 21st, 27th, and 48th hour during the precipitation process. From the 1st hour to the 48th hour, due to the effect of rainfall and infiltration, there is a certain uplift of the groundwater. Meanwhile, the pore water pressure in the upper stagnation area begin to rise at the 21 st hour, mainly concentrated at the junction of the perched water area and the eluvium. It increases further at the 27th hour and shows a significant difference at the 48th hour. Further change of the rainfall intensity and duration may result in an area with zero pore pressure in the perched water zone. 


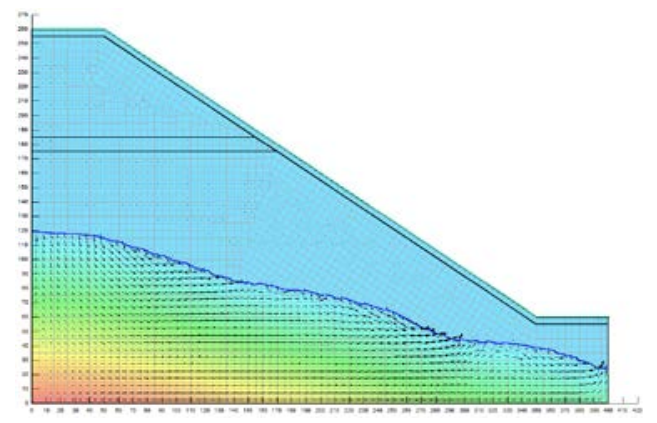

Fig. 7. At the 1st hour.

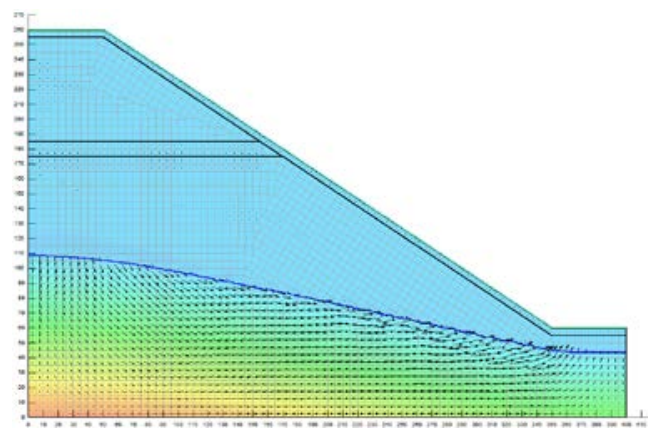

Fig. 9. At the 27 th hour.

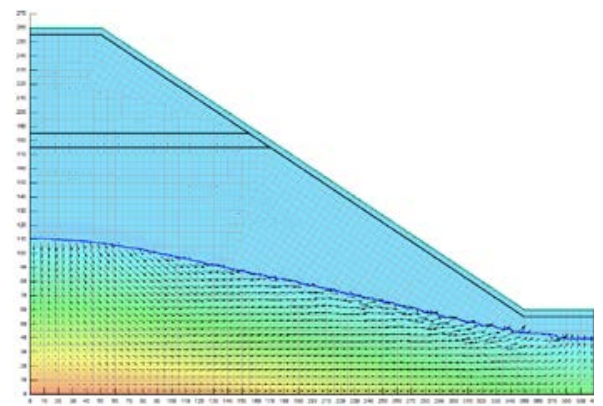

Fig. 8. At the 21 st hour.

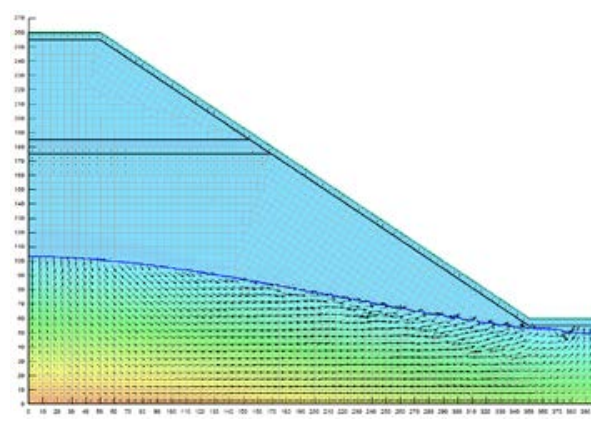

Fig. 10. At the 48th hour.

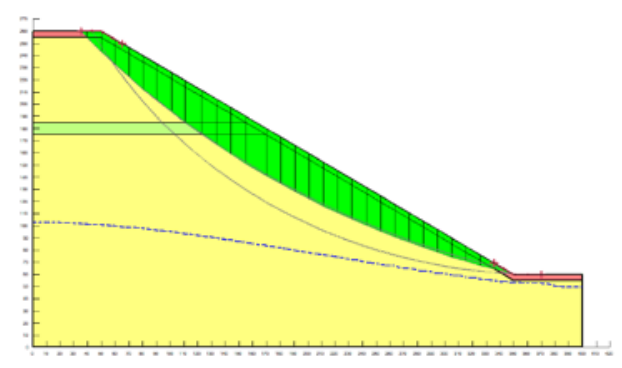

Fig. 11. Slope Stability at a Certain Moment.

Figure 11 shows the landslide situation at the 48th hour. The stability of slice is expressed by the safety factor. The smaller the safety factor, the lower the stability of the slice. The green part in the figure is the critical slice, that is, the slice with the minimum safety factor; and its safety factor is 0.743 . After 48 hours of precipitation, the safety factor decreases, which means that under the rainfall conditions, the risk of landslides is increased. The gray lines indicate other possible slip surfaces. The figure of safety factor and rainfall time shows that the landslide safety factor increases slightly in the early stage of less rainfall. However, after 21 hours and 27 hours of heavy rainfall, the safety factor of the landslide body shows a continuous trend of decline. As the rainfall decreases later, the landslide safety factor increases. Then after a long period of rainfall at an intensity of $10 \mathrm{~mm}$ per hour, the safety factor drops again. This proves that heavy rainfall, or prolonged rainfall of a certain intensity, increases the risk of landslides. 


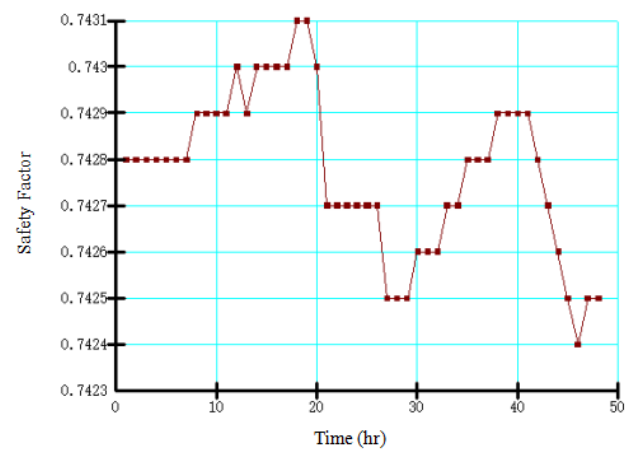

Fig. 12. Safety Factor Curve.

\section{Conclusion}

This paper is a study of the stability of a landslide in Sichuan Province. First, the Morgenstern-Price method was applied to analyze the stability of the landslide, then a simulation model was established in the GeoStudio software to analyze the stability of the landslide under complex conditions. By displaying the deformation, displacement and trend of geological disasters, this technology can provide methods and data for prevention and control for the government and reduce the loss of life and property caused by landslide hazards.

\section{Acknowledgments}

This work was supported by Science and Technology Project of State Grid Corporation of China (Contract No:SGSW0000AQJS2000094).

\section{References}

[1] Data on casualties are from conversations of D. K. Keefer with survivors..

[2] http://k.sina.com.cn/article_6145283913_16e499749020007e81.html

[3] Kai Y, Xuelian Y and Jun W 2018 Study on early warning of rainfall landslide area in mountainous highway Highway Engineering 43(06) 127-133

[4] Chimidi G, Raghuvanshi T K and Suryabhagavan K V 2017 Landslide hazard evaluation and zonation in and around Gimbi town, western Ethiopia - a GIS-based statistical approach Applied Geomatics 9(4)

[5] Fellenius W 1927 Erdstatische Bevechnungen (Berlin: W Ernstund Schu) p103

[6] Lam L and Fredlund D G 1994 A general limit equilibrium model for three dimensional slope stability analysis Can Geotech J 31 795-796

[7] Morgenstern N R and Price V 1965 The analysis of stability of general slip surface Geotechnique 15 79-93

[8] Shikai L, Zhongkai D and Li Z 2018 Genetic mechanism analysis and prevention of landslide in yaonan village, xinping County, yunnan province Yunnan geology 37(01) 99-102

[9] 2016 Stratigraphic Guide of China (All China Commission of Stratigraphy, Geological Publishing House) p103 
[10] State Bureau of quality and technical supervision 1998 Classification and nomenclature schemes of the rocks and Classification and nomenclature schemes of sedimentary rocks (China Standards Press)

[11] Yadong W 1989 Engineering Fluid Mechanics (China Architecture and Building Press)

[12] Shixiong Tang 2014 Research on Stability of Collapse Body Landslide Base on Saturated and Unsaturated Seepage Flow Theory Changsha University of Science and Technology

[13] Yaoting L 2006 On the hydrogeological conditions of groundwater in the triassic system of sichuan basin Journal of Salt Lake Research 1

[14] Lijuan S and Dan Z 2013 Analysis on the groundwater distribution of sichuan Province China Agri-production News 000(048) 165-168 